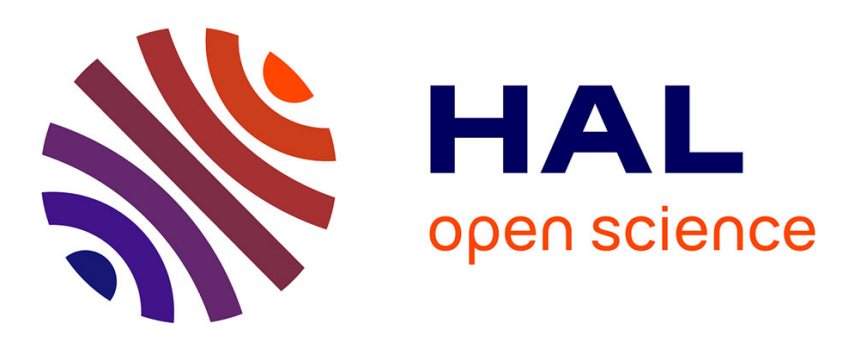

\title{
Introduction Os, sang, gènes, cultures
}

Caroline Costedoat, Stéphane Mazières

\section{To cite this version:}

Caroline Costedoat, Stéphane Mazières. Introduction Os, sang, gènes, cultures. C.N.R.S. Edition. Le sport-santé / Os, sangs, gènes et cultures, $N^{\circ} 17$ (1), pp.115, 2019, 9782271134837. 10.3917/corp1.017.0115. hal-02996073

\section{HAL Id: hal-02996073 https://hal.science/hal-02996073}

Submitted on 9 Nov 2020

HAL is a multi-disciplinary open access archive for the deposit and dissemination of scientific research documents, whether they are published or not. The documents may come from teaching and research institutions in France or abroad, or from public or private research centers.
L'archive ouverte pluridisciplinaire HAL, est destinée au dépôt et à la diffusion de documents scientifiques de niveau recherche, publiés ou non, émanant des établissements d'enseignement et de recherche français ou étrangers, des laboratoires publics ou privés. 


\section{Revue interdisciplinaire}

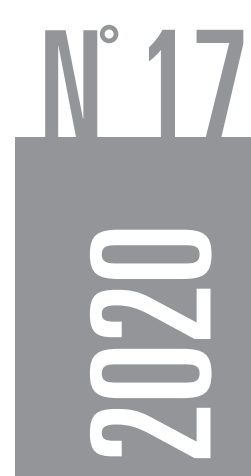

\section{Le sport-santé}




\title{
Sommaire
}

\author{
LIRE (1) \\ LE SPORT-SANTÉ \\ Dossier dirigé par Brice Favier-Ambrosini, Bernard Andrieu, Cécile Collinet
}

La santé en corps

Brice FAVIER-AMBRosINI

Partie 1. Gouvernement des corps et sport-santé

Activer sa vivacité : pour un sport-santé capacitaire.

Bernard ANDRIEU

Les pratiques de marche des Français : des profils variés

Jérémy Pierre, Cécile Collinet, Matthieu Delalandre

De la complexité des modèles normatifs à l'œuvre dans les

Réseaux Sport-Santé Bien-être

Brice FAVIER-AMBROSINI

\section{Partie 2 : Politiques publiquesdu sport-santé}

Les associations sportives au service des politiques de santé publique : les ressorts d'un engagement par la libre conformation

Marina Honta, Frédéric ILLIVI

Activités physiques et santé : une conversion des corps en marche..........

Gilles VIEILLE MarCHISET

Action publique et injonction du « sport-santé » :

les fédérations sportives, nouvelles opératrices de santé (1980-2018)...

Yves Morales 
Partie 3 : Trajectoires des patients dans les dispositifs d'incitationaux activités physiques et sportives dans un but de santé

Des patient.e.s face aux actions de promotion de la santé par les activités physiques : pluralité des formes de réception

Sandrine KNOBÉ

Activité physique adaptée et enjeux normatifs dans les trajectoires de maladie chronique aux points de rencontre des approches interactionnistes et de la philosophie de Georges Canguilhem

Claire PERRIN, Jean-Christophe MINo

\section{LIRE (2) \\ OS, SANGS, GÈNES ET CULTURES}

Dossier dirigé par Caroline Costedoat et Stéphane Mazières

Introduction. Os, sang, gènes, cultures

Caroline COSTEDOAT, Stéphane MAzIÈRES

ADN ancien et évolution humaine : nouveaux outils, nouvelles perspectives

Caroline Costedoat, Stéphane MazIÈRES

Diego, Asie et Amérique : comment la répartition d'un seul groupe sanguin suffit à décrire le peuplement de deux continents

Stéphane Mazières, Caroline COSTEDOAT

La transition néolithique : la contribution de la génétique Jacques Chiaroni, Aurore SCHMitT

Le génome humain : l'encre invisible (mais indélébile) de notre histoire.

Hugo NAYA, LUCía SPANGENBERG

La diaspora africaine dans les Amériques : l'apport des nouvelles approches génomiques pour illustrer l'héritage génétique des descendants africains dans le contexte de la traite négrière transatlantique. 
Le peuplement humain de Madagascar et son histoire : synthèse et hypothèses.

Harilanto RAZAFINDRAZAKA

L'identification individuelle à partir d'ADN et d'ossements : les dessous du travail de la police scientifique

Alain Stevanovitch

Paléopathologie et génétique : approches moléculaires pour la recherche et la caractérisation de microorganismes pathogènes à partir de restes humains

Avril Mefreay, Yann Ardagna, Philippe BIAgINI

\section{VOIR \\ L'EXPÉRIENCE DE LA CONSCIENCE CORPORELLE}

Petrucia Terezinha Da Nóbrega

L’Expérience de la Conscience Corporelle.

Petrucia Terezinha Da Nóbrega

\section{DÉCOUVRIR}

Migraine Buddy : Le Machine Learning au service de la santé

Marie Agostinucci

Obésité et image du corps Quelles attitudes face au regard de l'Autre?.

Laurence Tibère, Anne Dupuy, Jean-Pierre PoulaIN

DOSSIER 1

Dirigé par Sylvain Ferez et Philippe Liotard

Du génie du sport gay

Sylvain FEREZ, Philippe LIOTARD

Pratique ludo-communautaire et/ou production d'une performance sportive gay ? Approche sociohistorique de la natation et du volley-ball LGBT à Lausanne.

Saif Eddine Amaid, Laurent Paccaud, Sylvain FEREZ 
Ouvertures et frontières paradoxales du sport LGBT, entre dynamiques de protection et stratégies d'exposition

Antoine LE BLANC

Les Gay Games 1968-2018.

Cinquante ans d'innovations pour un sport inclusif

Philippe LIOTARD

L'irruption du VIH/sida dans l'espace sportif (1987-1997).

Enjeux socio-imaginaires d'une sécurisation par l'hygiène

Sylvain FEREZ

Les pratiquantes de Roller Derby et le jeu du genre

Fabienne Laval, Olivier SIROST

\section{DOSSIER 2}

Dirigé par Éric Perera, Anne Marcellini, Marius Matchuchescu, David Nocca

Contrôler sa silhouette : l'obésité face aux pressions sociales du recours aux techniques (biomédicales) de modification du corps

Éric Perera, Ante Marcelini, Marius Matchuchescu, David Nocca

Un corps « sur mesure » en « seulement » 30 minutes ? Monographie d'un centre de remise en forme réservé aux femmes

Cindy LOUCHET

L’obésité et la lutte contre le poids : enjeux médicaux et sociaux...............

Andrea SAGNI

Transformation de la silhouette à travers le grossissement des fesses en Côte d'Ivoire : normes sociales, représentations et enjeux sociaux.... Jean-Louis LogNon, Armelle Tania ANDOH 


\section{Introduction Os, sang, gènes, cultures}

Caroline COSTEDOAT

Stéphane MAZIĖRES

Quand la plupart de gens pense à l'anthropologie, l'image qui leur vient couramment à l'esprit est celle d'un intrépide aventurier (à la " Indiana Jones ») voyageant dans des lieux exotiques, travaillant courageusement dans n'importe quelles conditions, étudiant les caractéristiques sociales, symboliques, imaginaires et les pratiques des populations sur leurs terrains, ou encore extrayant des fossiles, des outils en pierre, ou d'autres évidences du passé, et faisant la une des journaux en proclamant avoir trouvé le chainon manquant de l'évolution humaine. Il y a de ça... mais pas que! Nous nous focaliserons dans ce dossier sur l'une des facettes de l'anthropologie.

De façon plus académique donc, l'Anthropologie biologique étudie la spécificité et la diversité humaine actuelle (variabilité) ou passée (histoire évolutive). Riche aujourd'hui d'un large panel d'outils et de matériels d'étude ce champ disciplinaire évolue. Les progrès technologiques associés à la mise en place d'approches interdisciplinaires ont permis l'étude de sources différentes, toutes plus riches les unes que les autres. Différentes facettes d'un prisme qui permettent une vision plus globale et plus précise de l'histoire. Elle se situe donc à l'interface de la biologie et des sciences humaines et intègre les apports d'autres disciplines (comme la biologie, l'anatomie, la génétique et la biologie moléculaire, la biodémographie, la médecine, les statistiques, l'histoire, la linguistique, l'ethnologie...). Cette discipline est aujourd'hui subdivisée en " sousspécialités » : génétique des populations, biologie humaine (variabilité humaine actuelle et processus d'adaptation à l'environnement et aux conditions pathogènes), paléoanthropologie (étude des populations du passé à partir de matériel fossile principalement os et dents), anthropologie funéraire, primatologie (étude des primates non humains), écologie humaine, biodémographie, anthropologie médico-légale... Elle doit être distinguée de l'anthropologie sociale et culturelle qui se rapproche de l'ethnologie ou parfois de la sociologie. L'anthropobiologie connaît actuellement une deuxième jeunesse parallèlement aux 
progrès techniques dans les domaines de la génétique, de l'imagerie, de l'informatique et des bio statistiques. Il parait impossible d'en établir de manière exhaustive un historique tant ses racines sont multiples et profondes au sein des sciences biologiques et humaines.

Le dossier que nous présentons " os, sang, gènes, cultures » est composé de huit articles qui vont nous permettre de présenter l'anthropologie dite évolutive à travers différentes dimensions diachroniques et synchroniques, à l'interface du biologique et du culturel.
Les techniques de génétiques moléculaires et les données qu'elles produisent ont la capacité de nous informer sur de nombreux aspects de notre histoire évolutive. Les analyses moléculaires peuvent éclairer la phylogénie, l'histoire démographique, l'histoire des forces sélectives, et même nous donner des informations sur l'anatomie et la physiologie des individus étudiés qui ne sont pas conservées directement dans les tissus durs des fossiles. Nous avons voulu, au travers de ce dossier, illustrer ces différentes facettes.

\section{Pourquoi ce titre de dossier : " os, sang, gènes, cultures »?}

Os. Cet « objet » d'étude à travers les prismes qu'il propose est l'essence même de la paléoanthropologie, de l'anthropologie funéraire, de la bioarchéologie. L'os est une véritable archive biologique, qui, par son étude, nous livre une multitude d'informations sur l'individu auquel il a participé, sur ses conditions de vie, sur l'état sanitaire, sur le patrimoine biologique de cet individu. Les séquences d'ADN de restes humains vieux de milliers d'années introduisent une nouvelle dimension historique dans la recherche des origines et de notre passé. À travers leurs études il est possible de remonter le cours de l'histoire.

Sang. Liquide à la fois éboueur, nourricier et pourvoyeur de vie, le sang est un tissu privilégié. Facile à prélever, il est composé d'éléments qui ont permis de comprendre le concept de pathologie moléculaire, charnière entre biologie et médecine. Il est aussi caractérisé par la permanence individuelle (de la naissance à la mort et au-delà) de certains caractères sanguins qui se transmettent de génération en génération selon les lois génétiques mendéliennes. Il est donc possible de reconnaitre, voire de définir, les populations. Enfin, le sang est « miroir de l'écologie » (Bernard, 1983), une véritable archive biologique sensible à l'environnement, aux modes de vie, autorisant la mise en évidence de précieuses corrélations biologie/environnement. Ainsi, en glissant le long des latitudes, des longitudes au travers des générations et des siècles, les biologistes, 
explorateurs des groupes sanguins, ont également rencontré l'histoire. L'étude du sang est donc devenue une méthode, un instrument de travail au service des anthropologues et des historiens. Méthode et instrument dont on peut apprécier la précision, la profondeur, l'aptitude à étudier le temps et l'espace.

Gènes. Dans une perspective de plus en plus microscopique et devenant de plus en plus difficile à appréhender sans une technologie adéquate, les gènes, eux aussi sont de véritables archives biologiques. La richesse des marqueurs génétiques réside dans leurs différences, leurs complémentarités. Il est ainsi possible d'obtenir des informations sur différentes fenêtres temporelles et spatiales. Les anthropologues et les généticiens parcourent le monde entier à la recherche d'échantillons d'ADN, égrainent les populations, décrivent leurs diversités. L'étude de la répartition géographique des polymorphismes populationnels permet ensuite d'interpréter ces patrons de diversité génétique, entre dérive, peuplement et sélection. Certains de ces marqueurs génétiques autorisent même une identification individuelle par comparaison de profils génétiques. Régi par un code universel, l'ADN est un véritable métronome biologique. Son étude a conduit à la découverte d'une saga d'une immense ampleur, une cartographie de l'histoire humaine ; une histoire de groupes, une histoire individuelle.

Cultures. Notre histoire ne peut se réduire à l'étude des composantes biologiques. Elle a aussi une dimension sociale, culturelle. Dans le terme " culture » nous entreposons une complexité de croyances, de valeurs, de comportements, de traditions, associés à une population donnée qui ont été acquis individuellement par imitation, par transmission, par apprentissage. On peut citer : le développement des langues, des processus de domestications, des religions, comme exemples parmi la quantité incroyable d'éléments culturels. Malgré la disparité qu'ils représentent, tous ces éléments influencent (ou ont influencé) notre diversité biologique. Le premier facteur connu pour être responsable de l'acquisition de " culture » par l'homme est notre système cognitif, irrémédiablement dérivé de notre génome... Ainsi, ces deux volets « biologiques » et « culturels » sont étroitement liés et en perpétuelles interactions. La prise en compte de cette dimension culturelle permet donc de mieux appréhender notre histoire.

\section{Pourquoi aujourd'hui une emphase sur l'ADN?}

L'ADN est aujourd'hui l'objet de toutes les attentions. Outil puissant mais non miraculeux, la crainte est de donner à cette molécule plus de pouvoir qu'elle ne peut en avoir. Il est vrai cependant que depuis quelques années, cet outil 
est rentré de façon massive dans les laboratoires, depuis les laboratoires de recherches biomédicales aux laboratoires d'écologie, depuis les sciences de la santé aux sciences de l'environnement. C'est donc naturellement qu'il est arrivé aux portes de l'anthropologie. Nous voyons aujourd'hui émerger des technologies génétiques complexes dont les pouvoirs, fascinants et redoutables sont popularisés dans les médias. Les dernières avancées techniques en matière d'ADN ancien ont amené ce domaine à l'avant-garde de la recherche en bioarchéologie. Bousculant ainsi une discipline et amenant la pipette au côté de la truelle dans la caisse de fouille des anthropologues. Bien qu'elles soient généralement fructueuses, les relations entre les anthropologues funéraires et les paléogénéticiens sont encore difficiles. Elles ne le sont pas moins entre les anthropologues médicaux-légaux et les généticiens, ces derniers travaillant à la mise en place de " portraits robots par étude ADN ». On peut s'interroger sur les raisons de telles peurs. La première raison vient s'en doute du fait que l'outil génétique n'est pas un outil " naturel » pour certaines disciplines et son utilisation, qui requiert des connaissances biologiques spécifiques sur le génome, n'est pas aisée. Nous sommes de fait au croisement entre les sciences humaines et les sciences dures.

Tous les laboratoires n'ont pas l'infrastructure pour extraire l'ADN ni les puissances et compétences informatiques pour traiter les données. De ce fait, les chercheurs font aujourd'hui appel à des entreprises privées ou semi-privées pour obtenir des informations sur la diversité génétique de leurs échantillons. Se pose alors la question de savoir que deviennent ces données ensuite ? Qui a le droit de les utiliser ? Dans quel cadre ? Quelle est la fiabilité des résultats ? Cet outil tellement à la mode devient incontournable dans les programmes de recherche car son utilisation permet souvent d'acquérir des financements... Or, comme nous l'avons dit précédemment et comme nous essayerons de le montrer au travers des différents articles, l'ADN n'est qu'un outil de l'anthropologie biologique d'aujourd'hui, au même titre que fut le pied à coulisse dans l'anthropologie physique de jadis, il permet de mesurer des homologies (ressemblance issue d'un ancêtre commun), des différences. Il semble que l'ADN devienne dans l'opinion un objet mythique, celui du salut d'une recherche anthropobiologique digne de ce nom, garant d'une scientificité ; objet de convoitises d'ordre non scientifique mais idéologique. D'une part, certaines problématiques de recherche ne nécessitent pas l'utilisation de cet outil pour répondre à leurs questions et son utilisation, sa sur-utilisation devient parfois aberrante.

D'autre part, les dérives potentielles qui découlent de la catégorisation des hommes et des femmes (le plus souvent à partir de critères phénotypiques déterminés par la génétique), ne viennent pas de l'utilisation de l'outil mais de ce que les individus (souvent non chercheurs mais politiques) font dire à ces données. 
Vous pouvez aujourd'hui, dans certains pays, donner de votre ADN pour avoir des informations sur votre " ancestry", connaître la part de Néandertal que vous avez dans votre génome ou encore estimer la probabilité que vous déclariez un cancer dans votre vie... La marchandisation et la médiatisation de l'outil génétique lui font beaucoup de torts. Les avancées technologiques et méthodologiques suscitent des questionnements éthiques qui ne sont pas toujours encadrés par des lois ; ou, si des lois existent, celles-ci sont différentes entre les pays. Ce décalage contribue à cette peur sociétale qui éclabousse également le monde de l'anthropologie. On imagine bien les dérives potentielles qui peuvent surgir de la mauvaise interprétation des données de variabilités génétiques. Cependant, il est aujourd'hui impensable de se priver de ce formidable outil.
Nous invitons donc à plonger dans la lecture de ce dossier. Il s'agit alors de prendre toute la mesure des potentialités et des limites de l'outil génétique, qui est un outil à multifacettes. Entre génome nucléaire et génome mitochondrial, entre différents types de marqueurs génétiques, chacun ayant son mode de transmission, sa vitesse d'évolution...un voyage est possible. Des caractéristiques leur permettant de répondre à différentes problématiques anthropologiques pourront être appréciées à la lecture de ces huit articles.

\section{Bibliographie}

Bernard J. 1983, Le Sang et l'histoire, Paris, Buchet-Chastel.

Caroline COSTEDOAT est Maître de conférences-HDR en génétique des populations à Aix- Marseille Université au sein de l'UMR ADES 7268 (AMU, CNRS, Établissement Français du Sang PACA Corse). Ces activités de recherches en anthropologie génétique se concentrent notamment sur l'étude des relations génome-environnement et sur des contextes d'identification populationnelle et individuelle du passé.

caroline.costedoat@univ-amu.fr

Stéphane MAZIERES est anthropologue généticien, Chargé de recherche au CNRS au laboratoire ADES, UMR7268 (Aix Marseille Université, CNRS, Etablissement Français du Sang PACA Corse). Ses travaux portent essentiellement sur l'étude de la diversité génétique humaine, la dynamique des peuplements, et l'étude de la répartition des polymorphismes du globule rouge entre les

populations.

stephane.mazieres@univ-amu.fr 